Research

Open Access

\title{
Premortem clinical diagnoses and postmortem autopsy findings: discrepancies in critically ill cancer patients
}

\author{
Stephen M Pastores, Alina Dulu, Louis Voigt, Nina Raoof, Margarita Alicea and Neil A Halpern
}

Critical Care Medicine Service, Department of Anesthesiology and Critical Care Medicine, Memorial Sloan-Kettering Cancer Center, 1275 York

Avenue, New York, NY 10021, USA

Corresponding author: Stephen M Pastores, pastores@mskcc.org

Received: 5 Feb 2007 Revisions requested: 9 Feb 2007 Revisions received: 20 Mar 2007 Accepted: 20 Apr 2007 Published: 20 Apr 2007

Critical Care 2007, 11:R48 (doi:10.1186/cc5782)

This article is online at: http://ccforum.com/content/11/2/R48

(C) 2007 Pastores et al.; licensee BioMed Central Ltd.

This is an open access article distributed under the terms of the Creative Commons Attribution License (http://creativecommons.org/licenses/by/2.0), which permits unrestricted use, distribution, and reproduction in any medium, provided the original work is properly cited.

\begin{abstract}
Introduction Limited data are available regarding the relationship of premortem clinical diagnoses and postmortem autopsy findings in cancer patients who die in an oncologic intensive care unit (ICU). The purposes of this study were to compare the premortem clinical and postmortem diagnoses of cancer patients who died in the ICU and to analyze any discrepancies between them.

Methods This is a retrospective review of medical records and autopsy reports of all cancer patients who died in a medicalsurgical ICU and had an autopsy performed between 1 January 1999 and 30 September 2005 at a tertiary care cancer center. Premortem clinical diagnoses were compared with the postmortem findings. Major missed diagnoses were identified and classified, according to the Goldman criteria, into class I and class II discrepancies.
\end{abstract}

Results Of 658 deaths in the ICU during the study period, 86 (13\%) autopsies were performed. Of the 86 patients, $22(26 \%)$ had 25 major missed diagnoses, 12 (54\%) patients had class I discrepancies, 7 (32\%) had class II discrepancies, and 3 (14\%) had both class I and class II discrepancies. Class I discrepancies were due to opportunistic infections (67\%) and cardiac complications (33\%), whereas class II discrepancies were due to cardiopulmonary complications (70\%) and opportunistic infections (30\%).

Conclusion There was a discrepancy rate of $26 \%$ between premortem clinical diagnoses and postmortem findings in cancer patients who died in a medical-surgical ICU at a tertiary care cancer center. Our findings underscore the need for enhanced surveillance, monitoring, and treatment of infections and cardiopulmonary disorders in critically ill cancer patients.

\section{Introduction}

Major discrepancy rates between premortem clinical diagnoses and postmortem autopsy findings continue to be reported in critically ill patients admitted to the intensive care unit (ICU) [1-22]. However, there are limited data regarding the relationship of premortem diagnoses and postmortem findings specifically in cancer patients who die in an oncologic ICU $[23,24]$. The purposes of this study were to compare the premortem clinical and postmortem diagnoses of cancer patients who died in the ICU and to analyze any discrepancies between them.

\section{Materials and methods}

This is a retrospective study of all patients who died in the ICU and had an autopsy performed between 1 January 1999 and 30 September 2005 at Memorial Sloan-Kettering Cancer
Center, a 435-bed tertiary care cancer center in New York City. The ICU is a 'closed' 12-bed adult medical-surgical unit staffed by anesthesiology and internal medicine housestaff, critical care fellows, and full-time critical care medicine attending physicians. The ICU attending physicians conduct multidisciplinary rounds twice daily and supervise and approve all clinical decisions in collaboration with the admitting medical and surgical teams. Our standard of care includes surveillance for nosocomial infections, aggressive and early use of broadspectrum antimicrobial agents in patients with suspected or proven infection, and routine use of antimicrobial-impregnated central venous catheters.

Consent for an autopsy is always requested from the health care proxy and/or a relative of the deceased patient by the ICU housestaff or attending physician and occasionally by the

$\overline{\mathrm{HSCT}}=$ hematopoietic stem cell transplantation; ICU = intensive care unit; LOS = length of stay. 
primary admitting team. Autopsies commonly are performed within 24 hours of death. The standard autopsy includes gross and histopathologic examination of all internal organs and the brain, when indicated.

All ICU admissions, deaths, and autopsies were identified by the hospital's institutional database. The following data were obtained from the electronic medical record for the ICU patients who died in the ICU and had autopsies: age, gender, admitting service (medical or surgical), underlying cancer diagnoses, and lengths of ICU stay and hospital stay. The major premortem clinical diagnoses and causes of death, including the immediate cause of death and the underlying primary disease, were recorded. The autopsy diagnoses were obtained from the final autopsy reports. Based on a review of the medical record and the autopsy diagnoses, two investigators ( $A D$ and $L V$ ) independently identified the clinical causes of death and then compared their results. If there was disagreement, the medical records were reviewed together by both investigators and a consensus on the cause of death was reached after discussion.

Discrepancies between premortem clinical and autopsy diagnoses were classified using the Goldman criteria (Table 1) [25]. For the purposes of this study, we focused only on the class I and class II major discrepancies [2]. Class I discrepancies were defined as a missed major diagnosis that, had it been made, would have changed management and might have resulted in prolonged survival. Class II discrepancies were a missed major diagnosis with no impact on treatment and survival because either the patient was already receiving appropriate therapy even though the diagnosis was not known or effective therapy was not available at the time.

Patients were categorized into three groups: (a) patients in whom a major clinical diagnosis was missed premortem (discordant cases), (b) patients in whom the premortem clinical diagnosis was confirmed on autopsy (concordant cases), and (c) patients in whom no pathologic diagnosis could be confirmed on autopsy.

\section{Statistical analysis}

Data are presented as means \pm standard deviations, absolute numbers, or percentages. Statistical analyses used included
Fisher exact test and one-way analysis of variance to test for differences among the three groups. $P$ values of less than 0.05 were considered significant. All statistical analyses were performed using statistical software (SPSS 12.0; SPSS Inc., Chicago, IL, USA). The study was approved by the institutional review board, which waived the need for informed consent.

\section{Results}

Between 1 January 1999 and 30 September 2005, 658 (20.2\%) of the 3,257 patients admitted to the ICU died. Of the 658 deaths, $86(13 \%)$ had an autopsy. During the study period, our autopsy rates averaged 13\% per year (range, 7.7\% to $21.2 \%$ per year).

Of the 86 patients who underwent an autopsy, 38 (44\%) were women and $48(56 \%)$ were men. The mean age was $54 \pm 16$ years. The mean length of stay (LOS) in the ICU was $9 \pm 8$ days, and the mean LOS in the hospital was $19 \pm 18$ days. Twenty-four patients (28\%) were surgical patients and 62 (72\%) were medical. Of the 24 surgical patients, 10 (42\%) underwent thoracotomy for lung or esophageal cancer, 10 (42\%) gastrointestinal/hepatobiliary surgery for hepatic or pancreatic cancer, 2 (8\%) orthopedic surgery for sarcoma, 1 head and neck cancer surgery, and 1 gynecologic cancer surgery. Of the 62 medical patients, 25 (40\%) had undergone hematopoietic stem cell transplantation (HSCT), 18 (29\%) had hematologic malignancies (leukemias or lymphomas), and 19 (31\%) had solid tumors.

Major missed diagnoses (discordant cases) were noted in 22 patients (26\%) (group 1): 12 (54\%) patients had class I discrepancies, 7 (32\%) had class II discrepancies, and 3 (14\%) had both class I and class II discrepancies. Among the 22 discordant cases, 6 had undergone surgery, 6 had hematologic malignancies, 6 had solid tumors, and 4 underwent HSCT.

Opportunistic infections were the most common class I discrepancies, followed by cardiac complications (thrombotic endocarditis, myocardial infarction, and heart failure) (Table 2). The opportunistic infections were due to one of several pathogens (viral, fungal, bacterial, and parasitic). The lung was the most commonly infected site, with pneumonia and empyema

Table 1

Goldman criteria for autopsy discrepancies [25]

\begin{tabular}{llll} 
Major discrepancies & Class I & Missed major diagnosis with potential adverse impact on survival and that would have changed management \\
& Class II & Missed major diagnosis with no potential impact on survival and that would have not changed therapy \\
\hline Minor discrepancies & Class III & Missed minor diagnosis related to terminal disease but not related to the cause of death \\
& Class IV & Other missed minor diagnosis
\end{tabular}




\begin{tabular}{|c|c|c|c|}
\hline & & & $N$ \\
\hline \multirow{13}{*}{$\begin{array}{c}\text { Class I } \\
\text { discrepancies } \\
(n=15)\end{array}$} & Opportunistic & VRE pneumonia & 2 \\
\hline & infections & Legionella pneumonia & 1 \\
\hline & $(n=10)$ & PCP pneumonia & 1 \\
\hline & & Invasive aspergillosis & 1 \\
\hline & & Candida empyema & 1 \\
\hline & & VZV meningoencephalitis & 1 \\
\hline & & HSV esophagitis & 1 \\
\hline & & CMV pneumonia & 1 \\
\hline & & Disseminated necrotizing toxoplasmosis & 1 \\
\hline & Cardiac & Ischemic cardiomyopathy & 2 \\
\hline & complications & Thrombotic endocarditis & 2 \\
\hline & $(n=5)$ & & \\
\hline & & Congestive heart failure & 1 \\
\hline \multirow{7}{*}{$\begin{array}{c}\text { Class II } \\
\text { discrepancies } \\
(n=10)\end{array}$} & Cardiopulmonary & Pulmonary embolism & 4 \\
\hline & complications & & \\
\hline & $(n=7)$ & Thrombotic endocarditis & 2 \\
\hline & & Pulmonary hemorrhage & 1 \\
\hline & Opportunistic & Candidemia & 1 \\
\hline & infections $(n=3)$ & VRE meningitis & 1 \\
\hline & & CMV proctitis & 1 \\
\hline
\end{tabular}

CMV, cytomegalovirus; HSV, herpes simplex virus; PCP, pneumocystis carinii pneumonia; VRE, vancomycin-resistant enterococcus; VZV, varicella-zoster virus.

present in seven patients, followed by central nervous system infections (two patients), gastrointestinal infections (two patients), and widely disseminated disease (two patients). The majority of class II discrepancies were accounted for by cardiopulmonary complications $(n=7)$ attributed to pulmonary emboli and thrombotic endocarditis (Table 2).

Clinical diagnoses were confirmed by autopsy in 49 patients $(57 \%)$ (group 2). Most of the confirmed diagnoses were due to bacterial or fungal infections. Autopsy was inconclusive in 15 patients (17\%) (group 3). Of the 15 patients, 12 (80\%) were medical patients and $3(20 \%)$ were surgical. The majority of group 3 patients died of multiple organ failure and systemic inflammatory response of unknown etiology, and no specific cause of death could be discerned on autopsy. The autopsies of these patients showed diffuse alveolar damage in the lung and diffuse non-specific inflammatory response with scaring and fibrosis in other organs, and positive cultures were not obtained.
There were no statistically significant differences in age or gender between the patients who had missed major diagnoses (group 1) and those with autopsy confirmation of premortem clinical diagnoses (group 2) (Table 3). However, the patients with no pathologic diagnosis made on autopsy (group 3) had a significantly longer ICU LOS compared to those with autopsy confirmation of premortem clinical diagnoses ( $p=$ 0.05). Overall, patients with autopsy confirmation of premortem clinical diagnoses were not significantly different from those with missed diagnoses $(p=0.11)$.

\section{Discussion}

In this study, we found an overall discrepancy rate of $26 \%$ between the premortem clinical and autopsy diagnoses in cancer patients who died in a medical-surgical ICU at a tertiary cancer center. Our discrepancy rate of $26 \%$ is within the range of discrepancy rates (5\% to $32 \%)$ that have been reported for autopsies performed in the general adult ICU population [1-22]. To our knowledge, however, only two previ- 
Table 3

Characteristics of critically ill cancer patients who underwent autopsy

\begin{tabular}{|c|c|c|c|c|}
\hline & $\begin{array}{c}\text { Missed major diagnosis } \\
\qquad(n=22)\end{array}$ & $\begin{array}{l}\text { Clinical diagnosis confirmed } \\
\qquad(n=49)\end{array}$ & $\begin{array}{l}\text { No pathologic diagnosis made } \\
\qquad(n=15)\end{array}$ & $P$ value \\
\hline Gender (males/females) & $12: 10$ & $29: 20$ & $7: 8$ & 0.688 \\
\hline Age (years) & $59 \pm 12$ & $55 \pm 15$ & $50 \pm 19$ & 0.223 \\
\hline ICU LOS (days) & $7.5 \pm 7$ & $8 \pm 10$ & $15 \pm 14$ & 0.039 \\
\hline Hospital LOS (days) & $15 \pm 13$ & $18 \pm 16$ & $29 \pm 26$ & 0.054 \\
\hline
\end{tabular}

Data are presented as means \pm standard deviations, absolute numbers, or percentages. ICU, intensive care unit; LOS, length of stay.

ous autopsy studies have examined diagnostic discrepancy rates in cancer patients who died in the ICU [23,24]. Gerain and colleagues [23] reported a 59\% major discrepancy rate in a medical oncologic ICU population. Unlike in our findings, the majority of major discrepancies were due to complications of the cancer itself or its treatment (for example, non-cardiogenic pulmonary edema, acute hemorrhage, and pulmonary embolism) rather than infection. We ascribe the marked difference in the discrepancy rates between the study by Gerain and colleagues [23] and our study (59\% versus 26\%) to the type of cancer patient population studied (medical versus mixed medical-surgical) and to improved diagnostic techniques and therapeutic strategies in recent years. However, when we compare our findings in a select patient population (the HSCT subgroup) to a similar HSCT population study [24], we observed an almost comparable, low discrepancy rate $(16 \%$ versus $7 \%$ ).

Opportunistic infections accounted for the majority (67\%) of class I discrepancies. In contrast to previous studies that showed a predominance of fungal infections in immunocompromised patients $[1,23]$, the opportunistic infections in our study were represented by various pathogens (viral, fungal, and parasitic) (Table 2). We ascribe these findings to the increasing exposure of our patients to broad-spectrum antimicrobials that effect the terminal flora and promote the emergence of more virulent and resistant nosocomial infections $[26,27]$. Our findings reinforce the difficulty of diagnosing different infectious entities such as nosocomial pneumonia and fungal and viral infections in critically ill patients [23,24]. We suggest that novel microbiologic identification with non-culture techniques, including serologic tests, immunohistologic methods, polymerase chain reaction, and molecular-probing technologies, be introduced to aid in the rapid diagnosis of these virulent infections $[28,29]$.

In this study, we describe a category of patients (group $3, n=$ 15) who experienced prolonged ICU and hospital LOSs and had uncertain premortem diagnoses, and their autopsies were inconclusive, showing only non-specific, chronic inflammatory, and fibrotic changes in various organs, including the lung, kidney, and liver. These findings are not unexpected as it is well known that autopsies of patients who die after a prolonged period of resuscitation and support in the ICU typically report multiple organ failure as the primary cause of death regardless of the different primary diagnoses [30]. Thus, in our opinion, postmortem information may be similarly limited in providing a specific diagnosis of the cause of death in cancer patients who die after a prolonged ICU and hospital LOS (Table 3).

The $13 \%$ average yearly autopsy rate in our study is much lower than that of other published postmortem studies from adult ICUs [1-6,8,11-17]. We ascribe our lower ICU autopsy rate to one of three possibilities. First, in our center, the physician caring for the patient during hospitalization may differ from the outpatient physician who has a long-standing rapport with the family. Additionally, when the patient is admitted to the ICU, the critical care team assumes primary care. Thus, there may not be a single physician with a close enough relationship to the patient's next of kin at the time of death to obtain consent for an autopsy. Second, due to the frequent use of advanced high-tech investigative modalities available at our center, both physicians and family members may perceive that the autopsy will have a low yield. Third, when patients with advanced cancer die, physicians and family members often attribute the death to the expected complications of the malignancy. In this circumstance, it is perceived that an autopsy is unnecessary.

Our study has several limitations, including the retrospective study design and selection bias that may have occurred; physicians and family members of patients with premortem diagnostic uncertainty would have been more likely to pursue an autopsy than in cases in which all parties were certain of the diagnoses and the outcome was predictable. Similar to prior studies $[13,15]$, we were unable to fully account for all the premortem diagnostic investigations that were performed on all the autopsied patients. Nevertheless, we believe that our findings may be extrapolated to similar critically ill cancer patients treated in general ICUs.

\section{Conclusion}

Our study suggests that missed major diagnoses with potential impact on treatment and survival were noted in $26 \%$ of critically ill cancer patients admitted to an oncologic ICU. The missed major diagnoses were commonly due to opportunistic 
infections and cardiac complications. Our findings underscore the need for enhanced premorbid surveillance, monitoring, and treatment of infections and cardiopulmonary disorders in critically ill cancer patients. However, given the limitations of present-day microbiologic evaluation and treatment and cardiac imaging at the ICU bedside, the autopsy remains an invaluable tool for retrospective diagnostic understanding of difficult cases, medical education, and quality assurance.

\section{Key messages}

- Missed major diagnoses with potential impact on treatment and survival were noted in $26 \%$ of cancer patients admitted to an oncologic ICU.

- Opportunistic infections and cardiac complications were the most commonly missed major diagnoses.

- Our findings underscore the need for enhanced surveillance, monitoring, and treatment of infections and cardiopulmonary disorders in critically ill cancer patients.

\section{Competing interests}

The authors declare that they have no competing interests.

\section{Authors' contributions}

SMP, AD, and LV were responsible for the study design and data analysis. All authors were involved in drafting the manuscript and have full access to the data and take full responsibility for its integrity. All authors read and approved the final manuscript.

\section{Acknowledgements}

We thank Hao Zhang, research assistant, Department of Anesthesiology and Critical Care Medicine, and Elyn Riedel, biostatistician, Department of Epidemiology and Biostatistics, for assistance with the data analysis.

\section{References}

1. Nadrous HF, Afessa B, Pfeifer EA, Peters SG: The role of autopsy in the intensive care unit. Mayo Clin Proc 2003, 78:947-950.

2. Combes A, Mokhtari M, Couvelard A, Trouillet JL, Baudot J, Henin $\mathrm{D}$, Gibert C, Chastre J: Clinical and autopsy diagnoses in the intensive care unit: a prospective study. Arch Intern Med 2004, 164:389-392.

3. Roosen J, Frans E, Wilmer A, Knockaert DC, Bobbaers H: Comparison of premortem clinical diagnoses in critically ill patients and subsequent autopsy findings. Mayo Clin Proc 2000, 75:562-567.

4. Blosser SA, Zimmerman HE, Stauffer JL: Do autopsies of critically ill patients reveal important findings that were clinically undetected? Crit Care Med 1998, 26:1332-1336.

5. Fernandez-Segoviano P, Lazaro A, Esteban A, Rubio JM, Iruretagoyena JR: Autopsy as quality assurance in the intensive care unit. Crit Care Med 1988, 16:683-685.

6. Gut AL, Ferreira ALA, Montenegro MR: Autopsy: quality assurance in the ICU. Intensive Care Med 1999, 25:360-363.

7. Barendregt WB, de Boer HHM, Kubat K: Quality control in fatally injured patients: the value of the necropsy. Eur J Surg 1993, 159:9-13.

8. Mort T, Yeston N: The relationship of pre mortem diagnoses and post mortem findings in a surgical intensive care unit. Crit Care Med 1999, 27:299-303.
9. Berlot G, Dezzoni R, Viviani M, Silvestri L, Bussani R, Gullo A: Does the length of stay in the intensive care unit influence the diagnostic accuracy? A clinical-pathological study. Eur J Emerg Med 1999, 6:227-231.

10. Sharma BR, Gupta M, Harish D, Singh VP: Missed diagnoses in trauma patients vis-à-vis significance of autopsy. Injury 2005, 36:976-983

11. Tai DY, El-Bilbeisi H, Tewari S, Mascha EJ, Wiedemann HP, Arroliga AC: A study of consecutive autopsies in a medical ICU: a comparison of clinical cause of death and autopsy diagnosis. Chest 2001, 119:530-536

12. Twigg SJ, McCrirrick A, Sanderson PM: A comparison of postmortem findings with post hoc estimated clinical diagnoses of patients who die in a United Kingdom intensive care unit. Intensive Care Med 2001, 27:706-710.

13. Silfvast T, Takkunen O, Kolho E, Andersson LC, Rosenberg P: Characteristics of discrepancies between clinical and autopsy diagnoses in the intensive care unit: a 5-year review. Intensive Care Med 2003, 29:321-324.

14. Ong AW, Cohn SM, Cohn KA, Jaramillo DH, Parbhu R, McKenney MG Barquist ES, Bell MD: Unexpected findings in trauma patients dying in the intensive care unit: results of 153 consecutive autopsies. J Am Coll Surg 2002, 194:401-406.

15. Perkins GD, McAuley DF, Davies S, Gao F: Discrepancies between clinical and postmortem diagnoses in critically ill patients: an observational study. Crit Care 2003, 7:R129-132.

16. Dimopoulos G, Piagnerelli M, Berre J, Salmon I, Vincent JL: Postmortem examination in the intensive care unit: still useful? Intensive Care Med 2004, 30:2080-2085.

17. Magret-Iglesias M, Vidaur Tello L, Fernandez Olsina S, Garcia Fontgivell JF, Blazquez Vilas S, Alonso Rubio S, Diaz Santos E, Sirvent Calvera JJ, Rello J: Discrepancies between clinical and pathological diagnosis in a polyvalent intensive care service. Med Intensiva 2006, 30:95-100.

18. Combes A, Luyt CE, Trouillet JL, Chastre J: Is there still a role for autopsies in the intensive care unit? Clin Pulm Med 2006, 13:188-193.

19. Pastores SM, Halpern NA: Autopsies in the ICU: we still need them! Crit Care Med 1999, 27:235-236.

20. Podbregar M, Voga G, Krivec B, Skale R, Pareznik R, Gabrscek L: Should we confirm our clinical diagnostic certainty by autopsies? Intensive Care Med 2001, 27:1750-1755.

21. Shojania KG, Burton EC, McDonald KM, Goldman L: Changes in rates of autopsy-detected diagnostic errors over time: a systematic review. JAMA 2003, 289:2849-2856.

22. Maris C, Martin B, Creteur J, Remmelink M, Piagnerelli M, Salmon I, Vincent JL, Demetter P: Comparison of clinical and post-mortem findings in intensive care unit patients. Virchows Arch 2007, 450:329-333.

23. Gerain J, Sculier JP, Malengreaux A, Rykaert C, Themelin L: Causes of deaths in an oncologic intensive care unit: a clinical and pathological study of $\mathbf{3 4}$ autopsies. Eur J Cancer 1990, 26:377-381.

24. Al-Saidi F, Diaz-Granados N, Messner H, Herridge MS: Relationship between premortem and postmortem diagnoses in critically ill bone marrow transplantation patients. Crit Care Med 2002, 30:570-573.

25. Goldman L, Sayson R, Robbins S, Cohn LH, Bettmann M, Weisberg $\mathrm{M}$ : The value of the autopsy in three medical eras. $N$ Engl $J$ Med 1983, 308:1000-1005.

26. Crnich CJ, Safdar N, Maki DG: The role of the intensive care unit environment in the pathogenesis and prevention of ventilatorassociated pneumonia. Respir Care 2005, 50:813-836.

27. Safdar N, Maki DG: The commonality of risk factors for nosocomial colonization and infection with antimicrobial-resistant Staphylococcus aureus, enterococcus, gram-negative bacilli, Clostridium difficile, and Candida. Ann Intern Med 2002, 136:834-844.

28. Pryce TM, Palladino S, Price DM, Gardam DJ, Campbell PB, Christiansen KJ, Murray RJ: Rapid identification of fungal pathogens in BacT/ALERT, BACTEC, and BBL MGIT media using polymerase chain reaction and DNA sequencing of the internal transcribed spacer regions. Diagn Microbiol Infect Dis 2006, 54:289-297.

29. Buchheidt D, Hummel M, Schleiermacher D, Spiess B, Hehlmann R: Current molecular diagnostic approaches to systemic infec- 
Critical Care Vol 11 No 2 Pastores et al.

tions with aspergillus species in patients with hematological malignancies. Leuk Lymphoma 2004, 45:463-468.

30. Baue AE: Multiple, progressive, or sequential systems failure: a syndrome of the 1970s. Arch Surg 1975, 110:779-781. 